Article

\title{
Hirtinone, a Novel Cycloartane-Type Triterpene and Other Compounds from Trichilia hirta L. (Meliaceae)
}

\author{
Ivo José Curcino Vieira ${ }^{1, *}$, Otoniel de Aquino Azevedo ${ }^{2}$, Jucimar Jorgeane de Souza ${ }^{1}$, \\ Raimundo Braz-Filho ${ }^{1}$, Milena dos Santos Gonçalves ${ }^{1}$ and Marcelo Francisco de Araújo ${ }^{1}$ \\ 1 Laboratório de Ciências Químicas, Universidade Estadual do Norte Fluminense Darcy Ribeiro, \\ 28013-602, Campos dos Goytacazes, RJ, Brazil \\ 2 Centro Universitário São Camilo, Campus I, Rua São Camilo de Léllis 01, \\ 29304-910 Cachoeiro de Itapemirim, ES, Brazil \\ * Author to whom correspondence should be addressed; E-Mail: curcino@uenf.br; \\ Tel.: +55-22-2739-6712; Fax: +55-22-2739-7248.
}

Received: 20 December 2012; in revised form: 1 February 2013 / Accepted: 18 February 2013 / Published: 26 February 2013

\begin{abstract}
One novel triterpene cycloartane-type, named hirtinone (1), six protolimonoids nilocitin (2), dihydronilocitin B (3), melianone epimers (4) and (5), piscidinol A (6) and melianone lactone (7), one tertranortriterpenoid, hirtin (8), and one sesquiterpene, spathulenol (9), were identified in the fruits of Trichilia hirta. The structures were established by 1D and 2D NMR $\left({ }^{1} \mathrm{H}\right.$ and ${ }^{13} \mathrm{C}-\mathrm{NMR}, \mathrm{DEPTQ},{ }^{1} \mathrm{H}-{ }^{1} \mathrm{H}-\mathrm{COSY}$, ${ }^{1} \mathrm{H}-{ }^{1} \mathrm{H}-\mathrm{NOESY}, \mathrm{HSQC}$ and HMBC), high resolution mass spectroscopy (HR-ESI-MS) and infrared (IR) spectral data.
\end{abstract}

Keywords: Meliaceae; Trichilia hirta; terpenoids; NMR data

\section{Introduction}

The Meliaceae family has attracted such a great interest among phytochemists interested in bioproduction because of its very complex and diverse chemical structures and its biological activity, mainly against insects [1-4]. The Trichilia genus (Meliaceae) consists of about 230 species distributed throughout tropical America, which are recognized for their significant economic importance and high commercial value. Phytochemical studies have revealed that this genus is rich in terpenoids, including triterpenes, limonoids, steroids and other terpene derivatives [3-6]. Species of this genus have been 
also studied for their insecticidal activities and their isolated compounds revealed complex and interesting structures, including various limonoids [5,7,8]. The isolation and structural elucidation of the two novel limonoids from the fruits of T. hirta collected in Espírito Santo State, Brazil, was reported by Cortez et al. in 1992 [9].

In the present paper, we report an investigation of a hexane extract of fruits of a T. hirta specimen, which allowed us to characterize nine terpenoids, including a novel cycloartane-type triterpene named hirtinone (1), five protolimonoids: nilocitin (2) [10,11], dihydronilocitin B [10,11] (3), melianone epimers (4) and (5) [11,12], piscidinol A (6) [10,11,13] and melianone lactone (7) [11,12], the tertranortriterpenoid hirtin (8) [14] and the sequiterpene spathulenol (9) [15]. The structures were established by spectrometric techniques, mainly HRESIMS and 1D and 2D NMR, and comparative analysis with literature values. The structures of all the isolated compounds are shown in Figure 1.

Figure 1. Chemical structure of the compounds isolated from the fruits of $T$. hirta.
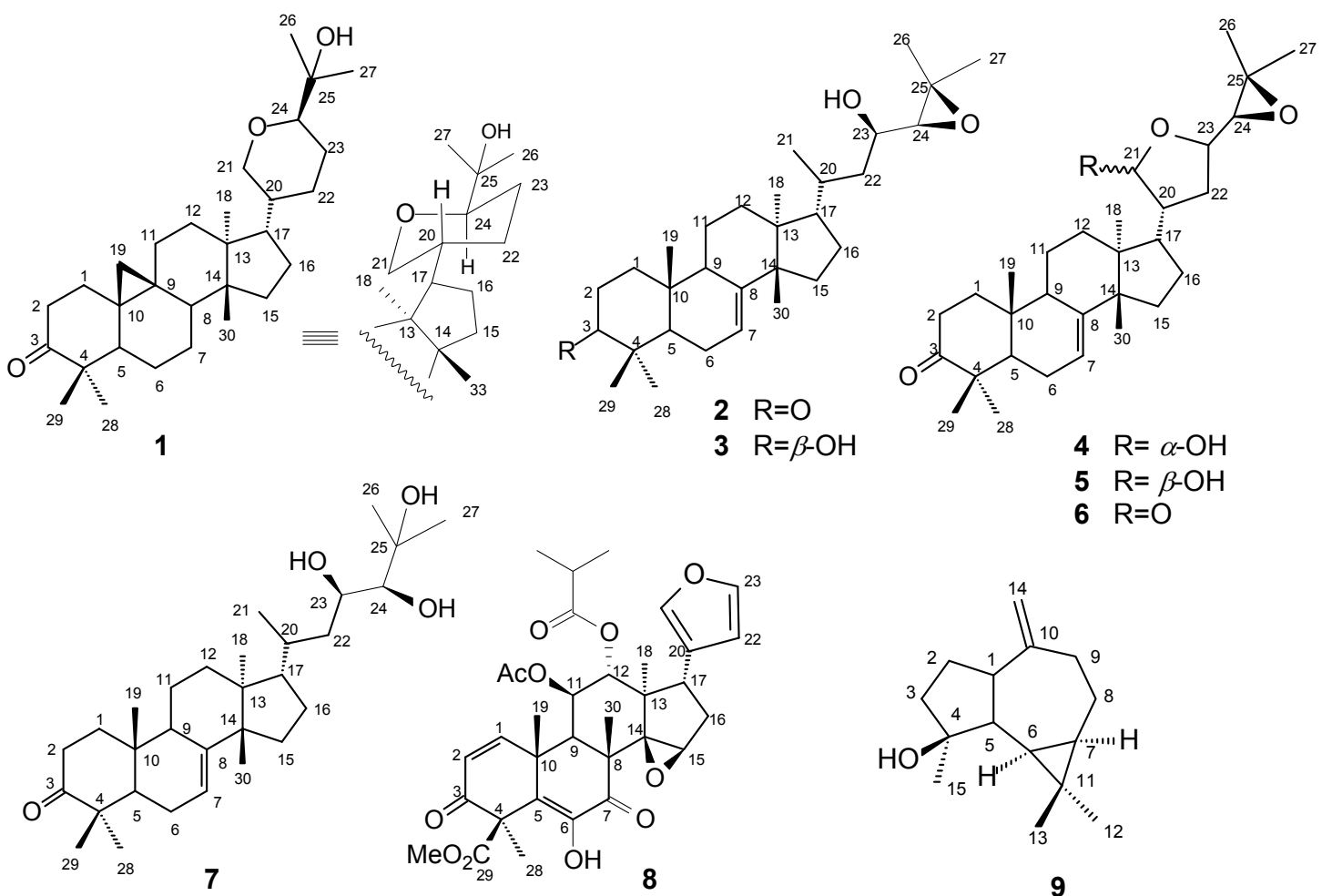

\section{Results and Discussion}

The crude hexane extract of T. hirta fruits was submitted to chromatography and furnished nine terpenoids 1-9. The known terpenoids, i.e., the six protolimonoids nilocitin (2), dihydronilocitin B (3), melianone epimers (4) and (5), piscidinol A (6) and melianone lactone (7), the tertranortriterpenoid, hirtin (8) and the sequiterpene spathulenol (9) were identified on the basis of ${ }^{1} \mathrm{H}$-and ${ }^{13} \mathrm{C}$-NMR spectral data, including ${ }^{1} \mathrm{H}-{ }^{1} \mathrm{H}-\mathrm{COSY},{ }^{1} \mathrm{H}-{ }^{1} \mathrm{H}-\mathrm{NOESY}$, HSQC and HMBC NMR experiments, which were also used to complete and unambiguous ${ }^{1} \mathrm{H}$ and ${ }^{13} \mathrm{C}$ chemical shift assignments $[16,17]$.

Hirtinone (1), m.p. $160-162{ }^{\circ} \mathrm{C}$, was isolated in an amorphous form. The IR spectrum showed bands at $v_{\max } 1,713$, characteristic of $\mathrm{C}=\mathrm{O}$ stretching of a ketone carbonyl group, as well as absorptions at $v_{\max }$ $3,354,2,925-2,852$ and $1,089 \mathrm{~cm}^{-1}$, characteristics of $\mathrm{OH}, \mathrm{C}-\mathrm{H}$ and $\mathrm{C}-\mathrm{O}$ stretching, respectively. 
The HR-ESI-MS spectrum of 1 utilizing the $\mathrm{ESI}^{+}$ionization mode showed a cationic base peak at $m / z 479.3485[\mathrm{M}+\mathrm{Na}]^{+}$, compatible with the molecular formula $\mathrm{C}_{30} \mathrm{H}_{48} \mathrm{O}_{3} \mathrm{Na}$ (calc. $\mathrm{m} / z$ 479.3501, $\left.\Delta_{m / z}=0.0016\right)$. These data and the comparative analysis of the $\left\{{ }^{1} \mathrm{H}\right\}$ - and DEPTQ- ${ }^{13} \mathrm{C}$ NMR spectra, that allowed us to identify signals (Table 1 ) corresponding to seven non-hydrogenated [including one $\mathrm{sp}^{2}$ of carbonyl group at $\delta_{\mathrm{C}} 216.6(\mathrm{C}-3)$ and one $\mathrm{sp}^{3}$ oxygenated at $\left.\delta_{\mathrm{C}} 71.6(\mathrm{C}-25)\right]$, five methine [all $\mathrm{sp}^{3}$ including one oxygenated at $\delta_{\mathrm{C}} 84.1(\mathrm{CH}-24)$ ], twelve methylene [all $\mathrm{sp}^{3}$ including one oxygenated at $\left.\delta_{\mathrm{C}} 72.8\left(\mathrm{CH}_{2}-21\right)\right]$ and six methyl carbon atoms, were used to propose the molecular formula $\mathrm{C}_{30} \mathrm{H}_{48} \mathrm{O}_{3}=(\mathrm{C}=\mathrm{O})(\mathrm{C}-\mathrm{O})(\mathrm{C})_{5}(\mathrm{O}-\mathrm{CH})(\mathrm{CH})_{4}\left(\mathrm{O}_{-}-\mathrm{CH}_{2}\right)\left(\mathrm{CH}_{2}\right)_{11}\left(\mathrm{CH}_{3}\right)_{6}$, seven unsaturation degrees (one carbonyl group in a triterpenoid with a cycloartane skeleton). The fragments postulated (Scheme 1) to justify the principal peaks observed in the HR-ESI-MS are compatible with a carbon skeleton of the cycloartane-triterpene type, including the presence of the cationized adduct as a complex involving two molecules of $\mathbf{1}$.

Scheme 1. Proposed fragmentation mechanisms of $\mathbf{1}$ (only peaks classified as principal ones, with intensity of the peaks (\%) in parentheses).

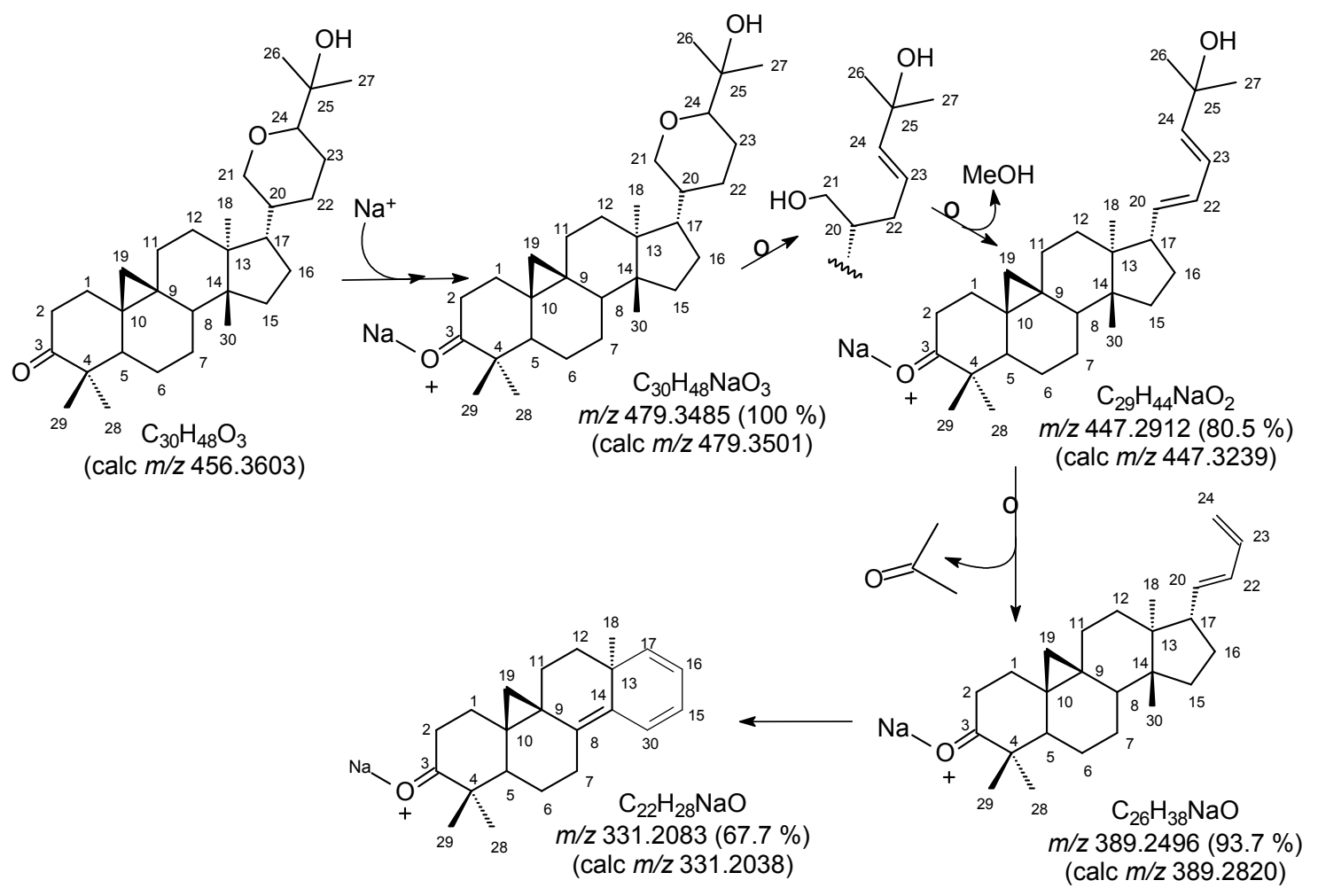

The ${ }^{1} \mathrm{H}-\mathrm{NMR}$ spectra (1D and ${ }^{1} \mathrm{H}-{ }^{1} \mathrm{H}-\mathrm{COSY}$, Table 1$)$ of $\mathbf{1}$, exhibiting a cycloartane triterpene profile, showed two doublets at $\delta_{\mathrm{H}} 0.82(J=4.1 \mathrm{~Hz})$ and $\delta_{\mathrm{H}} 0.62(J=4.1 \mathrm{~Hz})$, which were characteristic for a C-9/C-10 cyclopropyl methylene and singlet signals at $\delta_{\mathrm{H}} 0.98,1.19,1.16,1.12,1.08,1.07$ corresponding to six methyl groups. The presence of the signals at $\delta_{\mathrm{H}}(2.72 \mathrm{dt}, 6.4$ and $J=13.9 \mathrm{~Hz})$ and 2.34 (ddd, 1.7, 6.4 and $J=13.9 \mathrm{~Hz}$ ), correlated with the ${ }^{13} \mathrm{C}$ signal of a methylene carbon at $\delta_{\mathrm{C}}$ $37.5\left(\mathrm{CH}_{2}-2\right)$ in the HSQC spectrum indicated the presence of carbonyl group at carbon $\mathrm{C}-3$, characteristic of a cycloartan-3-one triterpenoid [18,19], which was confirmed by the HMBC spectrum (Table 1) with interactions between C-3 and the $3 \mathrm{H}-28,3 \mathrm{H}-29$ and $\mathrm{H}-5\left({ }^{3} J_{\mathrm{CH}}\right)$ as well as $\mathrm{H}-2\left({ }^{2} J_{\mathrm{H} \rightarrow \mathrm{C}}\right)$. 
Table 1. ${ }^{1} \mathrm{H}-(500 \mathrm{MHz})$ and ${ }^{13} \mathrm{C}-(125 \mathrm{MHz}) \mathrm{NMR}$ of hirtinone (1), including results obtained by heteronuclear 2D shift-correlated HSQC and $\mathrm{HMBC}$, in $\mathrm{CDCl}_{3}$ as solvent and TMS used as internal reference. Chemical shifts $(\delta, \mathrm{ppm})$ and coupling constants $(\mathrm{J}, \mathrm{Hz}$, in parenthesis) *.

\begin{tabular}{|c|c|c|c|c|c|}
\hline \multirow[b]{2}{*}{ Atom } & \multirow[b]{2}{*}{ Type } & \multicolumn{2}{|r|}{ HSQC } & \multicolumn{2}{|c|}{ HMBC } \\
\hline & & $\delta_{\mathrm{C}}$ & $\delta_{\mathrm{H}}$ & ${ }^{2} J_{H \rightarrow C}$ & ${ }^{3} J_{\mathrm{H} \rightarrow \mathrm{C}}$ \\
\hline 1 & $\mathrm{CH}_{2}$ & 33.4 & $1.87(\mathrm{~m}), 1.58(\mathrm{~m})$ & & $2 \mathrm{H}-19$ \\
\hline 2 & $\mathrm{CH}_{2}$ & 37.5 & $\begin{array}{c}2.72(\mathrm{dt}, 6.4 ; 13.9) \\
2.34(\mathrm{ddd} ; 1.7,6.4,13.9)\end{array}$ & & \\
\hline 3 & $\mathrm{C}$ & 216.6 & - & $\mathrm{H}-2 \mathrm{a}$ & 3Н-28; 3Н-29; H-5 \\
\hline 4 & $\mathrm{C}$ & 50.7 & - & $3 \mathrm{H}-28 ; 3 \mathrm{H}-29$ & \\
\hline 5 & $\mathrm{CH}$ & 48.6 & $1.73(\mathrm{~m})$ & & $2 \mathrm{H}-19 ; 3 \mathrm{H}-28 ; 3 \mathrm{H}-29$ \\
\hline 6 & $\mathrm{CH}_{2}$ & 26.4 & $2.06(\mathrm{~m}), 1.15(\mathrm{~m})$ & & \\
\hline 7 & $\mathrm{CH}_{2}$ & 21.5 & $1.60(\mathrm{~m}), 1.05(\mathrm{~m})$ & & \\
\hline 8 & $\mathrm{CH}$ & 49.4 & $1.54(\mathrm{~m})$ & & $3 \mathrm{H}-19 ; 3 \mathrm{H}-30$ \\
\hline 9 & $\mathrm{C}$ & 21.5 & - & $2 \mathrm{H}-19$ & \\
\hline 10 & $\mathrm{C}$ & 26.1 & - & $2 \mathrm{H}-19$ & \\
\hline 11 & $\mathrm{CH}_{2}$ & 26.1 & $1.80(\mathrm{~m}), 1.35(\mathrm{~m})$ & & $2 \mathrm{H}-19$ \\
\hline 12 & $\mathrm{CH}_{2}$ & 35.5 & $1.45(\mathrm{~m}), 1.30(\mathrm{~m})$ & & $3 \mathrm{H}-18$ \\
\hline 13 & $\mathrm{C}$ & 45.2 & - & $3 \mathrm{H}-18$ & $3 \mathrm{H}-30$ \\
\hline 14 & $\mathrm{C}$ & 48.6 & - & $3 \mathrm{H}-30$ & $3 \mathrm{H}-18$ \\
\hline 15 & $\mathrm{CH}_{2}$ & 32.2 & $1.60(\mathrm{~m})$ & & $3 \mathrm{H}-30$ \\
\hline 16 & $\mathrm{CH}_{2}$ & 26.6 & $1.40(\mathrm{~m}), 1.15(\mathrm{~m})$ & & \\
\hline 17 & $\mathrm{CH}$ & 47.9 & $1.62(\mathrm{~m})$ & & $3 \mathrm{H}-18$ \\
\hline 18 & $\mathrm{CH}_{3}$ & 18.5 & $0.98(\mathrm{~s})$ & & \\
\hline 19 & $\mathrm{CH}_{2}$ & 29.6 & $0.82(\mathrm{dl}, 4.1), 0.62(\mathrm{~d}, 4.1)$ & & \\
\hline 20 & $\mathrm{CH}$ & 39.2 & $1.55(\mathrm{~m})$ & & \\
\hline 21 & $\mathrm{CH}_{2}$ & 72.8 & $\begin{array}{c}4.21(\mathrm{dl}, 11.6) \\
3.06(\mathrm{t}, 11.6)\end{array}$ & & \\
\hline 22 & $\mathrm{CH}_{2}$ & 30.1 & $1.95(\mathrm{~m}), 1.10(\mathrm{~m})$ & & \\
\hline 23 & $\mathrm{CH}_{2}$ & 25.9 & $1.55(\mathrm{~m}), 1.38(\mathrm{~m})$ & & \\
\hline 24 & $\mathrm{CH}$ & 84.1 & $3.03(\mathrm{dd}, 11.4,2.0)$ & & $3 \mathrm{H}-26 ; 3 \mathrm{H}-27$ \\
\hline 25 & $\mathrm{C}$ & 71.6 & - & $3 \mathrm{H}-26 ; 3 \mathrm{H}-27$ & \\
\hline 26 & $\mathrm{CH}_{3}$ & 26.1 & $1.19(\mathrm{~s})$ & & $3 \mathrm{H}-22$ \\
\hline 27 & $\mathrm{CH}_{3}$ & 24.0 & $1.16(\mathrm{~s})$ & & $3 \mathrm{H}-26$ \\
\hline 28 & $\mathrm{CH}_{3}$ & 20.8 & $1.12(\mathrm{~s})$ & & $3 \mathrm{H}-24$ \\
\hline 29 & $\mathrm{CH}_{3}$ & 19.2 & $1.07(\mathrm{~s})$ & & $3 \mathrm{H}-28$ \\
\hline 30 & $\mathrm{CH}_{3}$ & 22.2 & $1.08(\mathrm{~s})$ & & \\
\hline
\end{tabular}

* Number of hydrogens bound to carbon atoms deduced by comparative analysis of HBBD- and DEPTQ $-{ }^{13} \mathrm{C}$ NMR spectra. Chemical shifts and coupling constants $(J)$ obtained from 1D ${ }^{1} \mathrm{H}-\mathrm{NMR}$ spectrum. Superimposed ${ }^{1} \mathrm{H}$ signals are described without multiplicity and chemical shifts deduced by HMQC, HMBC and ${ }^{1} \mathrm{H}-{ }^{1} \mathrm{H}-\mathrm{COSY}$ spectra.

The signals at $\delta_{\mathrm{H}} 4.21(\mathrm{dl}, 11.6 \mathrm{~Hz}, \mathrm{H}-21 \mathrm{eq}), 3.06(\mathrm{t}, 11.6 \mathrm{~Hz}, \mathrm{H}-21 \mathrm{ax})$ and 3.03 (dd, 11.4 and $2.0 \mathrm{~Hz}, \mathrm{H}-24)$ were attributed to hydrogen atoms of oxymethylene $\left(\mathrm{CH}_{2}-21\right)$ and oxymethine $(\mathrm{CH}-24)$, respectively, characteristic of the tetrahydropyran ring present in protolimonoid triterpenes isolated 
from Trichilia species [8,20]. The absorptions at $1713 \mathrm{~cm}^{-1}$ (carbonyl group), $1089 \mathrm{~cm}^{-1}$ (carbonoxygen bond) and $3354 \mathrm{~cm}^{-1}(\mathrm{OH})$ observed in the IR spectrum corroborate the NMR data. The coupling constant values $J=11.6 \mathrm{~Hz}$ and $J=11.4 \mathrm{~Hz}$ observed in the signals of hydrogens $\mathrm{H}-21 \mathrm{ax}$ (triplet at $\left.\delta_{\mathrm{H}} 3.06\right)$ and $\mathrm{H}-24\left(\mathrm{dd}\right.$ at $\delta_{\mathrm{H}} 3.03$ ) indicated an axial-axial interaction and, consequently, were used to define the axial positions of these H-20 and H-24 [20]. The analysis of the HSQC $\left({ }^{1} J_{\mathrm{H} \rightarrow \mathrm{C}}\right)$ experiment support the ${ }^{1} \mathrm{H}$ - and ${ }^{13} \mathrm{C}-\mathrm{NMR}$ data and led to assignment of six methyl signals at $\delta_{\mathrm{C}} / \delta_{\mathrm{H}}$ 18.5/1.07(s), 26.1/1.19(s), 24.0/1.16(s), 20.8/1.12(s), 19.2/0.98(s) and 22.2/1.08(s)] as well as signals of methylene $\mathrm{OCH}_{2}-21\left[\delta_{\mathrm{C}} / \delta_{\mathrm{H}} 72.8 / 4.21\right.$ and 3.06), methine $\mathrm{OCH}-24\left(\delta_{\mathrm{C}} / \delta_{\mathrm{H}} 84.1 / 3.03\right)$ and methylene $\mathrm{CH}_{2}-19\left(\delta_{\mathrm{C}} / \delta_{\mathrm{H}} 29.6 / 0.82\right.$ and 0.62$)$, which are compatible with the presence of tetrahydropyran ring [20] and cyclopropane ring in the compound 1 [18,19]. The presence of the cyclopropane ring was confirmed by HMBC spectrum analyses which showed cross-peaks 2H-19/C-9, 2H-19/C-10. The cross-peak at ${ }^{2} J_{\mathrm{H} \rightarrow \mathrm{C}} 3 \mathrm{H}-26 / \mathrm{C}-25$ and $3 \mathrm{H}-27 / \mathrm{C}-25$ supporting the proposal of hydroxyl group observed in the IR spectrum (Table 1).

The relative stereochemistry of $\mathbf{1}$ was determined from the coupling constants of relevant hydrogens, from the observed ${ }^{1} \mathrm{H}-{ }^{1} \mathrm{H}-\mathrm{NOESY}$ and from the comparison with data of analogous compounds with configuration described in the literature [20]. The relative stereochemistry of carbon atoms $\mathrm{CH}-20$ and $\mathrm{CH}-24$ (vide supra) was defined by coupling constants $J=11.6 \mathrm{~Hz}$ and $J=11.4 \mathrm{~Hz}$ observed in the signals of $\mathrm{H}-21 \mathrm{ax}$ (a triplet by coupling geminal with $\mathrm{H}-21 \mathrm{eq}-{ }^{2} J_{\mathrm{HH}}$ and vicinal with $\left.\mathrm{H}-20-{ }^{3} J_{\mathrm{HH}}\right)$ by and $\mathrm{H}-24{ }^{2} J_{\mathrm{HH}}$ with $\left.\mathrm{H}-23 \mathrm{ax}\right)$ and comparison with the literature ${ }^{13} \mathrm{C}-\mathrm{NMR}$ values of bourjotinolone A, a triterpene isolated from T. hispida [20], with both $\alpha$-orientations being consistent with the relative configuration shown in $\mathbf{1}$. Consistent with these observations, the ${ }^{1} \mathrm{H}-{ }^{1} \mathrm{H}-\mathrm{NOESY}$ spectrum of 1 showed cross-peaks assigned to dipolar interaction (spatial proximity). Thus, the structure of the new triterpene cycloartane-type, isolated from Trichilia hirta was established as 19-methylene-25-hydroxy-20,24-oxidecycloartan-3-one named hirtinone (1).

\section{Experimental}

\subsection{General Procedures}

Melting points were obtained on a Microquímica MQRPF and were uncorrected. FTIR spectra were recorded on a FTIR-8300 Shimadzu spectrometer using KBr disk. ESI-MS (high resolution) mass spectra were obtained by using a micrOTOF-QII (Bruker) mass spectrometer, using the positive ion mode of analysis. Chromatographic purifications were carried out by using silica gel $60(0.063-0.200 \mathrm{~mm})$.

${ }^{1} \mathrm{H}$ and ${ }^{13} \mathrm{C}$-NMR spectra were measured on a Bruker Utrashield 500 Plus spectrometer, operating at $500\left({ }^{1} \mathrm{H}\right)$ and $125\left({ }^{13} \mathrm{C}\right) \mathrm{MHz}$. $\mathrm{CDCl}_{3}$ was used as solvent with TMS as internal reference. Chemical shifts are given in the $\delta$ scale (ppm) and coupling constants $(J)$ in Hz. One dimensional (1D) ${ }^{1} \mathrm{H}$ and ${ }^{13} \mathrm{C}$-NMR spectra were acquired under standard conditions by using a direct detection $5 \mathrm{~mm}{ }^{1} \mathrm{H} /{ }^{13} \mathrm{C}$ dual probe. Standard pulse sequences were used for two dimensional spectra by using a multinuclear inverse detection $5 \mathrm{~mm}$ probe with field gradient. 


\subsection{Plant Material}

Fruits of Trichilia hirta were collected in May 2011, at Vale do Rio Doce Company, Linhares City, Espírito Santo State, Brazil. After botanical identification by botanist Domingos Folly. The voucher specimen of T. hirta was deposited at Vale do Rio Doce herbarium, under the code CRVD-6784.

\subsection{Extraction and Isolation}

Fruits of $T$. hirta were dried at room temperature until a constant weight was achieved (about one week). The dried and powdered fruits $(438.0 \mathrm{~g})$ were extracted with hexane (volume, time) at room temperature, furnishing, after solvent evaporation, $23.0 \mathrm{~g}$ of crude hexane extract. The hexane extract was chromatographed over a silica gel column with a gradient of hexane/ethyl acetate to afford nine fractions. Fraction 8 (1.36 g) was rechromatographed over a silica gel column with a gradient of hexane/acetone furnishing eighth fractions and $6(52.5 \mathrm{mg})$. Fractions $8.2(23.8 \mathrm{mg})$ and $8.3(524.2 \mathrm{mg})$ were rechromatographed over a silica gel column with a gradient of hexane/acetone yielding compounds $1(3.1 \mathrm{mg})$ and $\mathbf{9}(22.5 \mathrm{mg})$, respectively. Fraction $8.5(49.5 \mathrm{mg})$ was rechromatographed over a silica gel column with a gradient of hexane/acetone (8:2) $v / v$ furnishing compound 2 (4.5 mg). Fraction 8.7 (99.3 mg) was rechromatographed over a silica gel column with a gradient of hexane/acetone furnishing compound 3 (18.5 mg). Fraction 9 (4.03 g) was rechromatographed over a silica gel column with a gradient of hexane/acetone to provide compounds $7(67.0 \mathrm{mg}), \mathbf{4}+\mathbf{5}(176.0 \mathrm{mg})$ and $\mathbf{8}(65.7 \mathrm{mg})$.

Nilocitin (2): ${ }^{13} \mathrm{C} \delta$ (ppm): $38.56\left(\mathrm{CH}_{2}-1\right) ; 34.93\left(\mathrm{CH}_{2}-2\right) ; 216.96$ (C-3); 47.84 (C-4); $52.34(\mathrm{CH}-5)$; $24.36\left(\mathrm{CH}_{2}-6\right) ; 118.04$ (CH-7); 145.72 (C-8); 48.47 (CH-9); 36.05 (C-10); $18.86\left(\mathrm{CH}_{2}-11\right) ; 33.52$ $\left(\mathrm{CH}_{2}-12\right) ; 43.35$ (C-13); $51.23(\mathrm{C}-14) ; 34.06\left(\mathrm{CH}_{2}-15\right) ; 29.77\left(\mathrm{CH}_{2}-16\right) ; 53.28(\mathrm{CH}-17) ; 21.78$ $\left(\mathrm{CH}_{3}-18\right) ; 12.80\left(\mathrm{CH}_{3}-19\right) ; 33.69(\mathrm{CH}-20) ; 19.89\left(\mathrm{CH}_{3}-21\right) ; 40.47\left(\mathrm{CH}_{2}-22\right) ; 69.25(\mathrm{CH}-23) ; 68.46$ $(\mathrm{CH}-24) ; 60.32$ (C-25); $24.88\left(\mathrm{CH}_{3}-26\right) ; 19.82\left(\mathrm{CH}_{3}-27\right) ; 24.53\left(\mathrm{CH}_{3}-28\right) ; 21.61\left(\mathrm{CH}_{3}-29\right) ; 27.40$ $\left(\mathrm{CH}_{3}-30\right) .{ }^{1} \mathrm{H} \delta$ (ppm): (2.02; 1.50; 2H-1); (2.78dt 14.6, 5.6; 1H-2); (2.26 dt 14.6, 3.8; 1H-2) (1.70; $1 \mathrm{H}-5) ;(2.10 ; 2 \mathrm{H}-6)$; (5.33 m; 1H-7); (2.31; 1H-9); (1.60; 2H-11); (1.65; 1.40; 2H-12); (1.85; 1.55; 2H15); (2.08; 1.18; 2H-16); ( 1.50; 1H-17); (0.83 s; 3H-18); (1.03 s; 3H-19); (1.30; 1H-20); (0.98 s, 6.1; $3 \mathrm{H}-21) ;(1.70 ; 1.40 ; 2 \mathrm{H}-22) ;(3.61 \mathrm{~m} ; 1 \mathrm{H}-23) ;(2.68 d, 8.3 ; 1 \mathrm{H}-24) ;(1.35 s ; 3 \mathrm{H}-26) ;(1.34 s ; 3 \mathrm{H}-27)$; (1.07 $s ; 3 \mathrm{H}-28) ;(1.14 s ; 3 \mathrm{H}-29) ;(1.04 s ; 3 \mathrm{H}-30)$.

Dihydroniloctin (3): ${ }^{13} \mathrm{C} \delta$ (ppm): $37.19\left(\mathrm{CH}_{2}-1\right) ; 27.66\left(\mathrm{CH}_{2}-2\right) ; 79.25(\mathrm{CH}-3) ; 38.96(\mathrm{C}-4) ; 50.61$ (CH-5); $23.94\left(\mathrm{CH}_{2}-6\right) ; 118.05$ (CH-7); 145.57 (C-8); 48.91 (CH-9); 34.93 (C-10); $18.09\left(\mathrm{CH}_{2}-11\right)$; $33.97\left(\mathrm{CH}_{2}-12\right) ; 43.58$ (C-13); 51.79 (C-14); $33.78\left(\mathrm{CH}_{2}-15\right) ; 28.79\left(\mathrm{CH}_{2}-16\right) ; 53.25$ (CH-17); 13.11 $\left(\mathrm{CH}_{3}-18\right) ; 21.71\left(\mathrm{CH}_{3}-19\right) ; 33.59(\mathrm{CH}-20) ; 19.84\left(\mathrm{CH}_{3}-21\right) ; 40.69\left(\mathrm{CH}_{2}-22\right) ; 69.30(\mathrm{CH}-23) ; 68.51$ $(\mathrm{CH}-24) ; 60.34$ (C-25); $19.13\left(\mathrm{CH}_{3}-26\right) ; 24.88\left(\mathrm{CH}_{3}-27\right) ; 27.62\left(\mathrm{CH}_{3}-28\right) ; 14.71\left(\mathrm{CH}_{3}-29\right) ; 27.24$ $\left(\mathrm{CH}_{3}-30\right) .{ }^{1} \mathrm{H} \delta$ (ppm): (3.24 dd 11.1, 4.1; 1H-3); (5.26 sl; 1H-7); (0.75 s; 3H-18); (0.82 s; 3H-19); (0.96 d, 6.4; 3H-21); (3.57 m; 1H-23); (2.66 d, 8.2; 1H-24); (1.32 s; 3H-26); (1.33 s; 3H-27); (0.96s, $3 \mathrm{H}-28) ;(0.86 s ; 3 \mathrm{H}-29) ;(0.97 s ; 3 \mathrm{H}-30)$.

Melianone (4): ${ }^{13} \mathrm{C} \delta$ (ppm): $38.49\left(\mathrm{CH}_{2}-1\right) ; 34.91\left(\mathrm{CH}_{2}-2\right) ; 216.96(\mathrm{C}-3) ; 47.88(\mathrm{C}-4) ; 52.35(\mathrm{CH}-5)$; $24.38\left(\mathrm{CH}_{2}-6\right) ; 118.35$ (CH-7); 145.58 (C-8); 48.37 (CH-9); 35.10 (C-10); $17.72\left(\mathrm{CH}_{2}-11\right) ; 31.60$ 
$\left(\mathrm{CH}_{2}-12\right) ; 43.76$ (C-13); $51.03(\mathrm{C}-14) ; 33.93\left(\mathrm{CH}_{2}-15\right) ; 27.64\left(\mathrm{CH}_{2}-16\right) ; 49.37(\mathrm{CH}-17) ; 22.62\left(\mathrm{CH}_{3}-18\right)$; $12.75\left(\mathrm{CH}_{3}-19\right) ; 47.04(\mathrm{CH}-20) ; 101.19(\mathrm{CH}-21)$ e $97.74(\mathrm{CH}-21) ; 34.83\left(\mathrm{CH}_{2}-22\right) ; 77.50(\mathrm{CH}-23)$; 65.37 (CH-24); 57.30 (C-25); $24.92\left(\mathrm{CH}_{3}-26\right) ; 19.43\left(\mathrm{CH}_{3}-27\right) ; 24.44\left(\mathrm{CH}_{3}-28\right) ; 21.59\left(\mathrm{CH}_{3}-29\right)$; $27.28\left(\mathrm{CH}_{3}-30\right) .{ }^{1} \mathrm{H} \delta$ (ppm): (1.98; 1.45; 2H-1); (2.77 dt 14.5, 5.4; H-2); (2.25 dt 14.5, 4.0; H-2); (1.73; H-5); (2.15; 2H-6); (5.34; H-7); ( $2.30 \mathrm{H}-9) ;(1.62 ; 2 \mathrm{H}-11) ;(2.05 ; 1.75 ; 2 \mathrm{H}-12) ;(1.55 ; 2 \mathrm{H}-15)$; (1.95; 1.86; 2H-16); (1.82; H-17); (0.91 s; 3H-18); (1.02 s; 3H-19); (2.25; H-20); (5.38 dl 3.0; H-21); (2.15; 1.40; 2H-22); (3.95 m; H-23); (2.72 d 7.6; H-24); (1.34 s, 3H-26); (1.32 s; 3H-27); (1.06 $s$; $3 \mathrm{H}-28) ;(1.13 s ; 3 \mathrm{H}-29) ;(1.05 s ; 3 \mathrm{H}-30)$.

Piscidinol A (6): ${ }^{13} \mathrm{C} \delta$ (ppm): $38.25\left(\mathrm{CH}_{2}-1\right) ; 34.98\left(\mathrm{CH}_{2}-2\right) ; 47(\mathrm{C}-4) ; 52.31(\mathrm{CH}-5) ; 24.37\left(\mathrm{CH}_{2}-6\right)$; 117.95 (CH -7); 145 (C-8); 48.46 (CH-9); 35 (C-10); $18.31\left(\mathrm{CH}_{2}-11\right) ; 33.99$ (C-12); 43 (C-13); 51 (C-14); $33.77\left(\mathrm{CH}_{2}-15\right) ; 28.48\left(\mathrm{CH}_{2}-16\right) ; 53.60(\mathrm{CH}-17) ; 22.06\left(\mathrm{CH}_{3}-18\right) ; 12.80\left(\mathrm{CH}_{3}-19\right) ; 33.69(\mathrm{CH}-20)$; $18.92\left(\mathrm{CH}_{3}-21\right) ; 40.40\left(\mathrm{CH}_{2}-22\right) ; 69.71(\mathrm{CH}-23) ; 74.81$ (CH-24); 72 (C-25); $26.23\left(\mathrm{CH}_{3}-26\right) ; 27.50$ $\left(\mathrm{CH}_{3}-27\right) ; 24.39\left(\mathrm{CH}_{3}-28\right) ; 21.61\left(\mathrm{CH}_{3}-29\right) ; 27.41\left(\mathrm{CH}_{3}-30\right) .{ }^{1} \mathrm{H} \delta(\mathrm{ppm}):(2.05 ; 1.52 ; 2 \mathrm{H}-1) ;(2.78 d t$ 14.7, 5.7; H-2); (2.27 dt 14.7, 3.8; H-2); (1.75 t 8.9; H-5); (2.15 m; 2H-6); (5.34 tl 3.1; H-7); (2.40 m; H-9); (1.60; 2H-11); (1.85; 1.52; 2H-12); (1.70; 1.45; 2H-15); (1.90; 1.25; 2H-16); (1.71; H-17); (0.85 $s ; 3 \mathrm{H}-18) ;(1.03 s ; 3 \mathrm{H}-19) ;(1.48 ; \mathrm{H}-20)$; (0.96 d 6.4; 3H-21); (1.90; 1.25; 2H-22); (4.15 dd 9.7, 5.0; H-23); (3.10 sl; H-24); (1.36 s; 3H-26); (1.39 s; 3H-27); (1.07 s; 3H-28); (1.19 s; 3H-29); (1.04 s; 3H-30).

Melianone lactone (7): ${ }^{13} \mathrm{C} \delta$ (ppm): $38.51\left(\mathrm{CH}_{2}-1\right) ; 34.90\left(\mathrm{CH}_{2}-2\right) ; 216.76(\mathrm{C}-3) ; 47.88(\mathrm{C}-4) ; 52.57$ (CH-5); 24.39 ( $\left.\mathrm{CH}_{2}-6\right) ; 118.33$ (CH-7); 145.87 (C-8); 47.37 (CH-9); 35.12 (C-10); $17.64\left(\mathrm{CH}_{2}-11\right)$; $31.02\left(\mathrm{CH}_{2}-12\right) ; 43.91$ (C-13); 50.53 (C-14); $33.89\left(\mathrm{CH}_{2}-15\right) ; 24.22\left(\mathrm{CH}_{2}-16\right) ; 48.49$ (CH-17); 23.44 $\left(\mathrm{CH}_{3}-18\right) ; 12.73\left(\mathrm{CH}_{3}-19\right) ; 40.85$ (CH-20); 178.04 (C-21); $30.21\left(\mathrm{CH}_{2}-22\right) ; 77.96(\mathrm{CH}-23) ; 64.48$ (CH-24); 57.54 (C-25); $24.82\left(\mathrm{CH}_{3}-26\right) ; 19.47\left(\mathrm{CH}_{3}-27\right) ; 24.52\left(\mathrm{CH}_{3}-28\right) ; 21.55\left(\mathrm{CH}_{3}-29\right) ; 27.59\left(\mathrm{CH}_{3}-30\right){ }^{1} \mathrm{H} \delta$ (ppm): (2.10; 1.48; 2H-1); (2.60; 2.30; 2H-2); (1.51; H-3); (2.15; 1.95; 2H-6); (5.35 sl; H-7); (2.40; H-9); (1.60; 2H-11); (1.85; 1.75; 2H-12); (1.60; 2H-15); (1.90; 1.45; 2H-16); (2.17; H-17); (0.86 s; 2H-18); (1.05 s; 2H-19); (2.72; H-20); (2.35; 1.85; 2H-22); (4.18; H-23); (2.84; H-24); (1.40s; 2H-26); (1.37 s; $3 \mathrm{H}-27) ; 1.07 s ; 3 \mathrm{H}-28) ;(1.14 s ; 3 \mathrm{H}-29) ;(1.09 s ; 3 \mathrm{H}-30)$.

Hirtin (8): ${ }^{13} \mathrm{C} \delta(\mathrm{ppm}): 150.52(\mathrm{CH}-1) ; 125.84(\mathrm{CH}-2) ; 195.66(\mathrm{C}-3) ; 60.43(\mathrm{C}-4) ; 129.1(\mathrm{C}-5) ; 141.90$ (C-6); 196.19 (C-7); 46.15 (C-8); 42.01 (CH-9); 39.85 (C-10); 72.41 (CH-11); 77.76 (CH-12); 45.32 (C-13); 67.59 (C-14); 55.15 (CH-15); $32.15\left(\mathrm{CH}_{2}-16\right) ; 41.37$ (CH-17); $15.80\left(\mathrm{CH}_{3}-18\right) ; 25.97\left(\mathrm{CH}_{3}-19\right)$; 121.48 (C-20); 140.48 (CH-21); 111.22 (CH-22); 142.69 (CH-23); $22.85\left(\mathrm{CH}_{3}-28\right) ; 170.06$ (C-29); $22.52\left(\mathrm{CH}_{3}-30\right)$; $174.49\left(\mathrm{C}-1^{\prime}\right) ; 34.10\left(\mathrm{CH}-2^{\prime}\right) ; 18.70\left(\mathrm{MeO}-3^{\prime}\right) ; 18.68\left(\mathrm{MeO}-4^{\prime}\right) ; 169.39\left(\mathrm{C}-1^{\prime \prime}\right) ; 21.20$ (MeO-2"). ${ }^{1} \mathrm{H} \delta$ (ppm): (7.0 d 10.1; H-1); (6.19 d 10.1; H-2); (2.99 s; H-9); (5.37 s; H-11); (5.23 $\left.s ; \mathrm{H}-12\right)$; (2.93 s; H-15); (2.33 dd 13.8, 6.7; 2H-16); (2.02 dd 13.8, 11.0; 2H-16); (2.96 dd 11.0, 6.7; H-17); (0.84 $s$; 3H-18); (1.45 s; 3H-19); (7.15 sl; H-21); (6.2 sl; H-22); (7.32 t 1.6; H-23); (1.85 s; 3H-28); (2.47 sept 7.0; H-2'); (2.31 s; H2"-MeO); (1.10 d 7.0; H3'-MeO); $\left.0.98 d 7.0 ; \mathrm{H} 4^{\prime}-\mathrm{MeO}\right) ;(1.43 s ; 3 \mathrm{H}-30)$; $(6.47 s ; \mathrm{H}-\mathrm{HO})$.

Spathulenol (9): ${ }^{13} \mathrm{C} \delta$ (ppm): $53.42(\mathrm{CH}-1) ; 26,73\left(\mathrm{CH}_{2}-2\right) ; 41.74\left(\mathrm{CH}_{2}-3\right) ; 80(\mathrm{CH}-4) ; 54.33(\mathrm{CH}-5)$; 29.91 (CH-6); 27.48 (CH-7); $24.77\left(\mathrm{CH}_{2}-8\right) ; 38.86\left(\mathrm{CH}_{2}-9\right) ; 153,40$ (C-10); 20 (C-11); 16.15 $\left(\mathrm{CH}_{3}-12\right) ; 28.69\left(\mathrm{CH}_{3}-13\right) ; 108.28\left(\mathrm{CH}_{2}-14\right) ; 26.10\left(\mathrm{CH}_{3}-15\right) .{ }^{1} \mathrm{H} \delta$ (ppm): (2.22 m; H-1); $(2.08 \mathrm{~m}$; 
$1.90 m ; 2 \mathrm{H}-2) ;(1.80 m ; 1.60 m ; 2 \mathrm{H}-3) ;(1.30 t 3.5 ; \mathrm{H}-5) ;(0.49 \mathrm{dd}, J=11.3,9.5 \mathrm{~Hz}$; H-6); (0.74 m; H-7); (1.70 m; 2H-8); (2.4 dd, $J=12.3,5.3 \mathrm{~Hz}, 2 \mathrm{H}-9) ;(1.06 s, 3 \mathrm{H}-12) ;(1.08 \mathrm{~s}, 3 \mathrm{H}-13) ;(4.71 s ; 4.69 s ; 2 \mathrm{H}-14)$; $(1.32 s ; 3 \mathrm{H}-15)$.

\section{Conclusions}

The hexane extract from the fruits of $T$. hirta provided five protolimonoids $2-7$, one tertranortriterpenoid $\mathbf{8}$ and one sesquiterpene 9, which were isolated in a previous phytochemical investigation. A cycloartane-type triterpene 1, named hirtinone, is described for the first time in the literature.

\section{Supplementary Materials}

Supplementary materials can be accessed at: http://www.mdpi.com/1420-3049/18/3/2589/s1.

\section{Acknowledgments}

The authors are grateful to Fundação Carlos Chagas Filho de Amparo à Pesquisa do Rio de Janeiro (FAPERJ), Consenho Nacional de Desenvolvimento Científico e Tecnológico (CNPq) and Coordenação de Aperfeiçoamento de Pessoal de Nível Superior (CAPES) for scholarships and financial support.

\section{Conflict of Interest}

The authors declare no conflict of interest.

\section{References}

1. Pennington, T.D.; Styles, B.D. A generic monograph of Meliaceae. Blumea 1975, 22, 419-540.

2. Pupo, M.T.; Adorno, M.A.T.; Vieira, P.C.; Fernandes, J.B.; Silva, M.F.G.F.; Pirani, J.R. Terpenoids and steroids from Trichilia species. J. Braz. Chem. Soc. 2002, 13, 382-388.

3. Rodrigues, V.F.; Carmo, H.M.; Braz-Filho, R.; Mathias, L.; Vieira, I.J.C. Two new terpenoids from Trichilia quadrijuga (Meliaceae). Nat. Prod. Comm. 2010, 5, 179-184.

4. Vieira, I.J.C.; Figueiredo, E.R.; Freitas, V.R.; Mathias, L.; Braz-Filho, R.; Araújo, R.M. A new sesquiterpene from Trichilia casarettii (Meliaceae). Am. J. Anal. Chem. 2010, 2, 70-72.

5. Rodrigues, V.F.; Carmo, H.M.; Oliveira, R.R.; Braz-filho, R.; Mathias, L.; Vieira, I.J.C. Isolation of terpenoids from Trichilia quadrijuga (Meliaceae) by droplet counter-Current Chromatography. Chromatographia 2009, 70, 1191-1195.

6. Ramírez, M.C.; Toscano, R.A.; Arnason, J.; Omar, S.; Cerda-Garcia-Rojas, C.M.; Mata, R. Structure, conformation and absolute configuration of new antifeedant dolabellanes from Trichilia trifolia. Tetrahedron 2000, 56, 5085-5091.

7. Xie, Y.S.; Isman, M.B.; Gunning, P.; Mackinnon, S.; Arnason, J.T.; Taylor, D.R., Sánchez, P.; Hasbun, C.; Towers, G.H.N. Biological activity of extracts of Trichilia species and the limonoid hirtin against Lepidoptera larvae. Biochem. Syst. Ecol. 1994, 22, 129-136. 
8. Cortez, D.A.G.; Fernandes, J.B.; Vieira, P.C.; Silva, M.F.G.F.; Ferreira, A.G.; Cass, Q.B.; Pirani, J.R. Meliacin Butenolides from Trichilia estipulata. Phytochemistry 1998, 49, 2493-2496.

9. Cortez, D.A.G.; Vieira, P.C.; Fernandes, J.B.; Silva, M.F.G.F.; Ferreira, A.G. Limonoids from Trichilia hirta. Phytochemistry 1992, 31, 625-628.

10. Vieira, I.J.C.; Rodrigues-Filho, E.; Vieira, P.C.; Silva, M.F.G.F.; Fernandes, J.B. Quassinoids and protolimonoids from Simaba cedron. Fitoterapia 1998, LXIX, 88-90.

11. Itokawa, H.; Kishi, E.; Morita, H.; Takeya, K. Cytotoxic quassinoids and tirucallane-type triterpenes from the woods of Eurycoma longifolia. Chem. Pharm. Bull. 1992, 40, 1053-1055.

12. Biavatti, M.W.; Vieira, P.C.; Silva, M.F.G.F.; Fernandes, J.B.; Albuquerque, S. Triterpenoids constituents of Raulinoa echinata. J. Nat. Prod. 2002, 65, 562-565.

13. Gray, A.I.; Bhandari, P.; Waterman, P.G. New protolimonoids from the fruits of Phellodendron chinense. Phytochemistry 1988, 27, 1805-1808.

14. Simmonds, M.S.J.; Stevenson, P.C.; Porter, E.A.; Veitch, N.C. Insect antifeedant activity of three new tetranortriterpenoids from Trichilia pallida. J. Nat. Prod. 2001, 64, 1117-1120.

15. Ferreira, M.J.P.; Constantin, M.B.; Sartorelli, P.; Rodrigues, G.V.; Limberger, R.; Henriques, A.T.; Kato, M.J.; Emerenciano, V.P. Computer-aided method for identification of components in essential oil by ${ }^{13} \mathrm{C}-\mathrm{NMR}$ spectroscopy. Anal. Chim. Acta 2001, 447, 125-134.

16. Claridge, T.D.W. High-Resolution NMR Techniques in Organic Chemistry; Pergamon: Amsterdam, The Netherland, 1999.

17. Bretmaier, E.; Voelter, W. Carbon-13 NMR Spectroscopy: High-Resolution Methods and Applications in organic Chemistry and Biochemistry, 3rd ed.; VCH Publisher: Weinheim, Germany, 1987.

18. Furlan, M.; Lopes, M.N.; Fernandes, J.B.; Pirani, J.R. Diterpenes from Guarea trichilioides. Phytochemistry 1996, 41, 1159-1161.

19. Hisham, A.; Ajitha Bai, M.D.; JayaKumar, G.; Nair, M.S.; Fujimoto, Y. Triterpenoids from Dysoxylum malabaricum. Phytochemistry 2001, 56, 331-334.

20. Jolad, S.D.; Hoffmann, J.J.; Cole, J.R. Constituents of Trichilia hispida (Meliaceae). 2. A New Triterpenoid, Hispidone, and Bourjotinolone A. J. Org. Chem. 1980, 45, 3132-3135.

Sample Availability: Samples of the compounds 1-9 are available from the authors.

(C) 2013 by the authors; licensee MDPI, Basel, Switzerland. This article is an open access article distributed under the terms and conditions of the Creative Commons Attribution license (http://creativecommons.org/licenses/by/3.0/). 